\title{
Effects of an internet-based cognitive behavioral therapy (iCBT) intervention on improving depressive symptoms and work- related outcomes among nurses in Japan: a protocol for a randomized controlled trial
}

\author{
Kazuto Kuribayashi ${ }^{1 *}$ (D, Kotaro Imamura², Kazuhiro Watanabe², Yuki Miyamoto ${ }^{1}$, Ayumi Takano ${ }^{3}$, Utako Sawada',
} Natsu Sasaki ${ }^{2}$, Mariko Suga', Atsushi Sugino', Yui Hidaka ${ }^{2}$, Mako lida ${ }^{1}$, Mie Sudo ${ }^{1}$, Masahito Tokita ${ }^{4}$ and Norito Kawakami

\begin{abstract}
Background: Depression is a major problem among nurses; hence, it is important to develop a primary prevention strategy to manage depression among nurses. This randomized controlled trial (RCT) study aims to investigate the effects of a newly developed internet-based cognitive behavioral therapy (iCBT) program on depressive symptoms, measured at baseline and three- and six-month follow-ups, among nurses in Japan.
\end{abstract}

Methods: Nurses working at three university hospitals, one public hospital, and twelve private hospitals who meet inclusion criteria will be recruited and randomized either to the intervention group or the control group (planned N=525 for each group). The newly developed iCBT program for nurses consists of six modules, which cover different components of cognitive behavioral therapy (CBT); transactional stress model (in module 1), self-monitoring skills (in module 2), behavioral activation skills (in module 3), cognitive restructuring skills (in modules 4 and 5), relaxation skills (in module 5), and problem-solving skills (in module 6). Participants in the intervention group will be asked to read these modules within 9 weeks. The primary outcome will be depressive symptoms as assessed by the Beck Depression Inventory-II (BDI-II) at baseline, three-, and six-month follow-ups.

Discussion: The greatest strength of this study is that it is the first RCT to test the effectiveness of the iCBT program in improving depressive symptoms among nurses. A major limitation is that all measurements, including major depressive episodes, are self-reported and may be affected by situational factors at work and participants' perceptions.

Trial registration: This trial was registered at the University Hospital Medical Information Network clinical trials registry (UMIN-CTR; ID = UMIN000033521) (Date of registration: August 1, 2018).

Keywords: Internet-based, Cognitive behavioral therapy, Stress, Depression, Stress management, Prevention

\footnotetext{
* Correspondence: kkuribayashi-jans@umin.ac.jp

'Department of Psychiatric Nursing, Graduate School of Medicine, The

University of Tokyo, 7-3-1 Hongo, Bunkyo-ku, Tokyo 113-0033, Japan

Full list of author information is available at the end of the article
}

(c) The Author(s). 2019 Open Access This article is distributed under the terms of the Creative Commons Attribution 4.0 International License (http://creativecommons.org/licenses/by/4.0/) which permits unrestricted use, distribution, and reproduction in any medium, provided you give appropriate credit to the original author(s) and the source, provide a link to the Creative Commons license, and indicate if changes were made. The Creative Commons Public Domain Dedication waiver (http://creativecommons.org/publicdomain/zero/1.0/) applies to the data made available in this article, unless otherwise stated. 


\section{Background}

Most nurses work in stressful settings [1]. Work-related stress among nurses can result from various persistent sources, including heavy workloads, interpersonal conflicts, the emotional impacts of care, lack of reward or control, and shift work [2]. Occupational stress is considered a leading risk factor for a variety of negative health outcomes, including depression [2]. Depression is a major global public health problem due to its high prevalence worldwide and debilitating effects [3]. Nurses can be more vulnerable to depression due to a lack of stress-management skills and/or organizational factors at work [4]. Two surveys conducted in the U.S. reported that the prevalence rate of depression among nurses was $18-35 \%$, higher than among the general population $[5,6]$. Depression has deleterious consequences not only on individuals' biopsychosocial states, but also on organizations' productivity and functionality [7]. Depression's biopsychosocial effects impact somatic symptoms [5], quality of life [6], absenteeism [8], work engagement [9], and intention to leave employment [8]. Within organizations, depression affects the quality of care nurses provide [10] and contributes to economic and productivity loss in the workplace [6]. It is essential to maintain nurses' mental health to ensure their individual well-being, the quality of patient care, and cost-effectiveness. As such, it is important to manage work-related stress and depression among nurses and adverse organizational factors impacting nurses at work as a primary approach to depression prevention.

Concerning stress management for workers in general, a meta-analytic review reported that cognitive behavioral therapy (CBT) interventions significantly reduced depressive or anxiety symptoms [11]. Similarly, two meta-analyses concluded that techniques based on CBT and relaxation were effective in reducing work-related stress among workers and that $\mathrm{CBT}$ and relaxation were more effective than other interventions $[12,13]$. The estimated effect sizes (Cohen's d) for CBT and relaxation on work-related stress were 0.681.16 and $0.35-0.50$, respectively $[12,13]$. In the nursing workplace, a Cochrane review reported that compared to no intervention, CBT and relaxation stress-management interventions significantly improved stress-related outcomes, including occupational stress and depressive symptoms, among nurses (standardized mean difference [SMD] $=-0.34$ and - 0.59, respectively, at six-month follow-up) [4].

With increased Internet penetration, a new delivery approach for CBT via the Internet has developed, called Internet-based CBT (iCBT). An iCBT program provides users the same principles and techniques as face-to-face CBT programs in a highly structured electronic format consisting of several educational lessons and homework assignments [14]. Benefits of iCBT include its high accessibility and confidentiality. Participants can access the program anytime, anywhere from various Internet-connected devices and review its contents and related feedback as often as they like, which can be useful for users [15]. A literature review stated that the merits of Internet-based interventions in the workplace include the removal of time and location limitations, allowing access to a larger target group at low cost, and increased participant confidentiality, thus reducing barriers to accessing mental health services [16]. The iCBT program could be appropriate for nurses in particular considering that most nurses work in rotating shifts, there are few suppliers of face-to-face CBT programs, like the Employee Assistance Program (EAP), in hospital settings [17], and few nurses receive face-to-face counseling because of barriers to seeking help for mental health $[2,18]$.

For the working population, stress management based on iCBT has been shown to reduce depressive symptoms. One meta-analysis showed that $\mathrm{iCBT}$ interventions had a significant effect on psychological well-being, reducing depressive symptoms $(g=0.25)$ and increasing work effectiveness, including engagement and productivity $(g=0.26)$, at post-intervention [19]. Another metaanalysis reported that iCBT programs had a small significant positive effect on workers' mental health conditions at post-intervention $(g=0.15)$ [20]. An iCBT intervention might also be effective for nurses in improving depressive symptoms and other work-related outcomes. However, only one randomized controlled trial (RCT) of $\mathrm{iCBT}$ has targeted nurses in the U.S. [21]. In that study, the iCBT intervention significantly improved occupational stress among hospital nurses, though its effect on depressive symptoms was not assessed [21]. Although depression has a debilitating effect on nurses, no study has evaluated the effects of an iCBT program on improving depressive symptoms. An RCT must be conducted to verify the effectiveness of an iCBT program in improving depressive symptoms among nurses. Since nurses in the U.S. might be exposed to very different work cultures and stressful situations, experience different thoughts and feelings, and demonstrate different behaviors and coping strategies compared to Japanese nurses, a new iCBT program must be developed for nurses in Japan.

\section{Objectives and hypotheses}

This study has two purposes: (1) to develop a new six-week iCBT program for nurses in Japan and (2) to examine the effectiveness of this iCBT program in improving depressive symptoms as the primary outcome and prevention of the onset of major depressive disorder and improvement in psychosocial work environment, work engagement, and work performance as the secondary outcomes at three- and sixmonth follow-ups among nurses in Japan.

We hypothesize that the iCBT program will: (1) be associated with lower depressive symptoms at three- and six-month follow-ups among nurses; (2) reduce the risk of major depressive episode (MDE) at the six-month 
follow-up, and (3) improve the psychosocial work environment, work engagement, and work performance at three- and six-month follow-ups.

\section{Methods}

\section{Trial design}

This study will be a two-arm parallel-group non-blinded RCT with three- and six-month follow-ups. Participants will be requested to complete a baseline online questionnaire. Subsequently, participants meeting the inclusion criteria will be randomly allocated either to the intervention or the control group at a 1:1 ratio. This study's iCBT program will be provided to the participants in the intervention group for 9 weeks. All surveys will be conducted using online questionnaires. Online follow-up surveys will be implemented at three (immediately after the intervention) and 6 months after the baseline survey. After the six-month follow-up, the iCBT program will be provided to participants in the control group. The study protocol was registered at the University Hospital Medical Information Network (UMIN) Clinical Trials Registry (UMIN-CTR, ID = UMIN000033521). This manuscript was written according to the Standard Protocol Items: Recommendations for Interventional Trials (SPIRIT) guidelines [22].

\section{Participants}

This study will target nurses (i.e., without mental illness) working in a hospital. The participants will be recruited from three university hospitals, one public hospital, and twelve private hospitals (The University of Tokyo Hospital, Yokohama City University Hospital, Yokohama City University Medical Center, Chiba Cerebral and Cardiovascular Center, IMS Katsushika Heart Center, Hokutokai Sawa Hospital, Hoikukai Yokohama Aihara Hospital, Seikeikai Komagino Hospital, Ichiyoukai Youwa Hospital, Hekisuikai Hasegawa Hospital, Inokashira Hospital, Tokyo Musashino Hospital, Sekizenkai Soga Hospital, Sakuunkai Iiduka Hospital, Sakuunkai Yuurin Hospital, and The Salvation Army Booth Memorial Hospital) in Japan. We will recruit nurses from all wards and outpatient sections.

All participants will be required to meet to following inclusion criteria:

1. Registered nurse.

2. Currently employed as a full-time employee by the hospital.

3. Can access the Internet via their own PC, smartphone, or tablet PC.

Participants will be excluded in the following cases:
1. Nurses who are going to take a leave of absence for any reason or leave the hospital during the sixmonth study period.

2. Practical nurse or nursing aide.

3. Non-regular, part-time, or temporary nurses.

4. Diagnosed with major depressive disorder during the previous month (assessed via the online version of the WHO-Composite International Diagnostic Interview (WHO-CIDI v 3.0) [23].

5. Diagnosed with lifetime bipolar disorder (WHOCIDI v 3.0).

6. Sick leave for 15 or more days in total due to own health problems during the past 3 months.

7. Those who currently receive treatment, including psychotherapy, from a mental-health professional.

8. Strong suicidal ideation (score of 3 on item no. 9 of the Beck Depression Inventory-II (BDI-II)).

\section{Procedure}

Figure 1 shows the participant flowchart for this trial. The researchers will email an invitation via the nursing departments to the nurses who work at three university hospitals, one public hospital, and twelve private hospitals. The email will include an outline of the study and the URL of a website that will fully describe the study. Candidates will click the URL and read a full explanation of the purpose and procedures of the study on the website. Nurses who are interested in participating will be asked to mark the consent option and type their name, the name of the hospital where they are working, and their email address to give their consent. This consent information will be sent to the research center and preserved. Nurses who are not interested in participating will be asked to close the website. Subsequently, participants who submitted the consent information will receive an email containing the URL of the baseline survey. They will click the URL and input their baseline information. After completing the baseline questionnaire, the participants who meet eligibility criteria will be randomized either to the intervention or to the control group. The researchers will inform the participants of their group assignment. In order to avoid delay between the randomization and the beginning of intervention, the researchers will assign the participants to a group as soon as the baseline survey is completed. The researchers will send an email containing the iCBT program's URL and an ID and password to the participants in the intervention group. Participants in the intervention group will click the URL, enter their own ID and password, and receive the iCBT program. They will be asked not to share their ID, password, and the iCBT program contents with others. Those in the control group will receive the iCBT program after the six-month follow-up. 


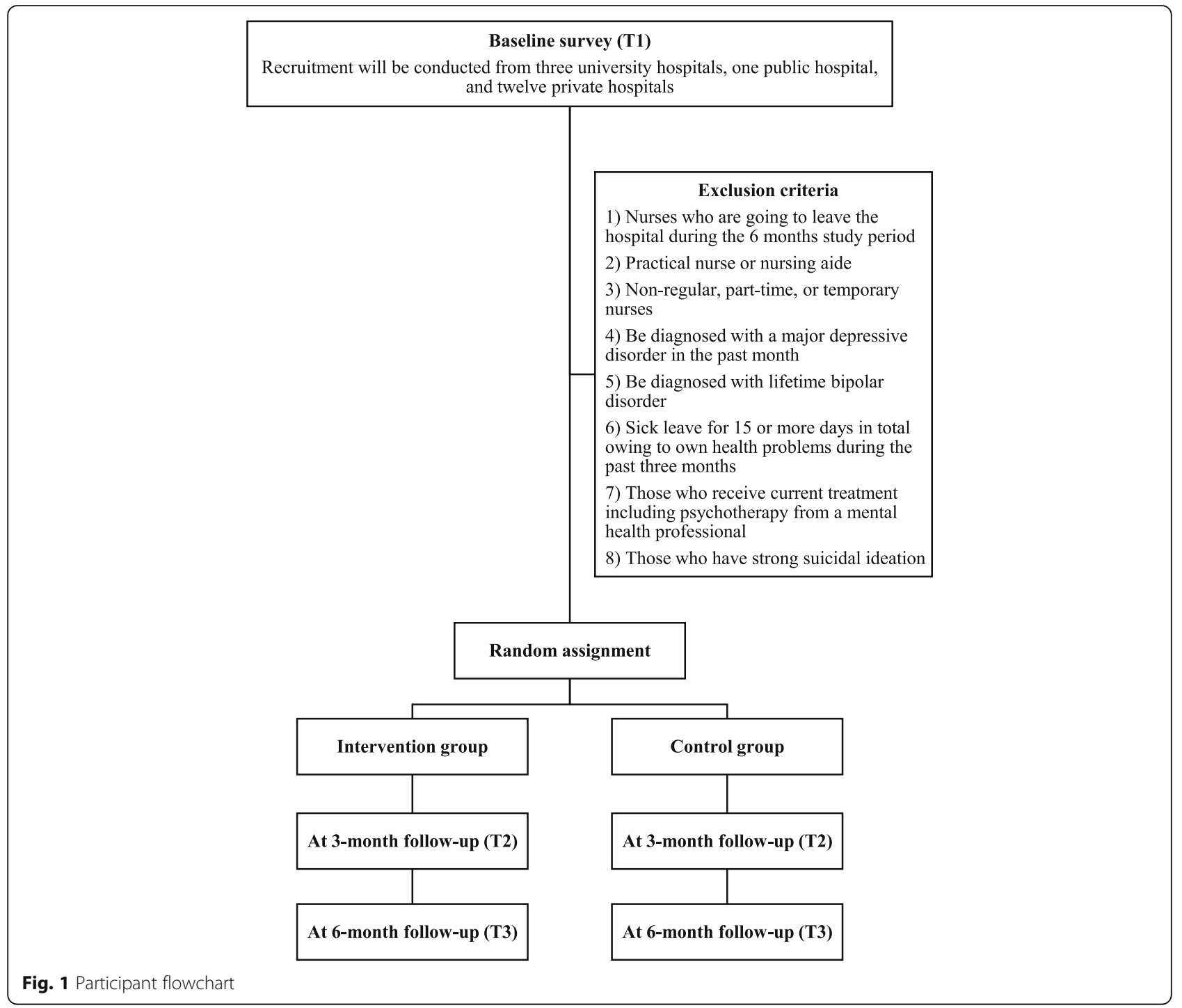

\section{Intervention program}

This study's iCBT program for nurses was developed by extensively modifying a previous $\mathrm{iCBT}$ program aimed at improving depressive symptoms among newly graduated nurses [24]. The iCBT program for newly graduated nurses was developed through extensive modification the iCBT program for workers developed by Imamura et al. (2014) [25]. To develop the iCBT program for newly graduated nurses, we interviewed experienced nurses working in various wards to identify the most common stressful situations they experienced as newly graduated nurses as well as their thoughts, feelings, behaviors, and coping strategies in such situations. These interviews yielded a number of common stressful situations-lack of confidence in new knowledge and skills, being scolded by senior nurses, difficulty in communicating with and reporting to a senior nurse, heavy workloads, and shift work-and associated distorted cognitive patterns. Similarly, in developing this study's iCBT program, the content had to reflect the normal work situations and culture experienced by nurses in Japan. Thus, hearings were conducted with nurses in Japan who were working in various wards and outpatient departments in hospitals as full-time employees to identify common stressful situations and nurses' thoughts, feelings, behaviors, physical feelings, and coping strategies in such situations. Based on these hearings, we modified case stories to reflect common and major stressors (i.e., when changing wards or entering a workplace, nurses face stressful situations, such as an unfamiliar environment, new knowledge and skill requirements, and interpersonal problems) and replaced one module (on communication assertiveness) with another (behavioral activation) because nurses acquire communication skills over time.

Participants allocated to the intervention group will study the iCBT program. Called "Useful mental health solutions for work and everyday life," the program is a 
six-module internet-based training program that provides stress-management skills based on CBT via a manga story. The use of manga in learning has been reported to 1) stimulate interest in learning, 2) facilitate understanding of the contents, and 3) maintain the motivation to learn [26, 27]. Some existing iCBT programs (e.g., THIS WAY UP [28]; SPARX [29]) utilize comics and manga characters to enhance understanding and users' motivation to learn the contents. This study's iCBT program will also use comics and manga characters to increase users' understanding and motivation. Each week, a new module will be delivered. At the end of each module, the participants will be asked to submit homework that will ask them to apply their learned skills to nursing or daily life situations that they personally perceive as stressful. This homework will be voluntary. Nurses will be able to complete and submit homework only on a PC or a tablet PC, as we lacked sufficient resources and funding to make this function available on smartphones. Each module will require about $30 \mathrm{~min}$ to complete, including homework. Participants can receive the program at any time and place via the Internet. Nurses in the intervention group will be asked to read the six modules within 9 weeks after the program starts. After 9 weeks, researchers will close all modules.

Table 1 presents the contents of this study's iCBT program. The iCBT program will consist of six modules, each covering a different component of CBT: transactional stress model (in module 1), self-monitoring skills (in module 2), behavioral activation skills (in module 3), cognitive restructuring skills (in module 4 and 5), relaxation skills (in module 5), and problem-solving skills (in module 6).

This study's iCBT program will contain two supplemental modules. One is the introduction module. When participants in the intervention group begin the program for the first time, they will be urged to complete the introduction module, which provides a general description of the program. The introduction module will consist of a brief description of stress and an explanation of the operability of this iCBT program. Participants will be informed about the negative effects of stress on physical and mental health. Furthermore, they will be shown how to use the internet-based program. Another module will contain a mood self-assessment using Kessler's Psychological Distress Scale (K6) [30, 31], which we call the "mood checker." Participants can assess their own psychological health at any time using this module. The detailed explanation of the operability and self-monitoring are likely to improve the attrition rate, a limitation of applying internet-based interventions [32, 33]. According to previous systematic reviews, the mean attrition rate in internet-based psychological intervention programs conducted in the workplace is $23 \%$, although it varies significantly across studies [19]. Low literacy in the use of internet-based programs and limited feedback regarding participants' status could lead to dropout [32-34]. Improving attrition rates in internet-based intervention programs increases the frequency with which users engage with the contents [19], which may be an important determinant of effectiveness [35].

Additionally, this iCBT program includes two supports. One is feedback support from researchers proficient in CBT on homework. Participants who submit their homework will receive feedback to encourage their understanding. Five researchers in the department of mental health and psychiatric nursing will provide feedback. To maintain high feedback quality, the researcher (KK) will offer simple instructions and training along with a manual that will include an example of feedback comments. Another is question support. Participants will be able to ask the researcher (KK) questions about the program or its contents. The researcher will answer the questions in a timely manner. Supported iCBT programs have been reported to be more effective in the management of depressive symptoms compared to self-guided iCBT programs $[15,20]$. Additionally, supported ehealth programs have been reported to have lower attrition rates than those without support [36]. The two-way supports offered by this program may enhance adherence to and the effectiveness of this program.

Table 1 Contents of the iCBT program for nurses

\begin{tabular}{lll}
\hline $\begin{array}{l}\text { Module } \\
\text { number }\end{array}$ & Title & Contents \\
\hline 1 & Mechanism of stress & $\begin{array}{l}\text { Learning about a transactional stress model and noticing stress responses and } \\
\text { stressors }\end{array}$ \\
2 & $\begin{array}{l}\text { Tips on self-case formulation using a CB } \\
\text { model }\end{array}$ & $\begin{array}{l}\text { Learning about a CB model and the tips for self-case formulation using this model } \\
3\end{array}$ \\
$\begin{array}{l}\text { Tips to increase your activity } \\
\text { Cognitive restructuring, part 1 }\end{array}$ & $\begin{array}{l}\text { Learning about behavioral activation skills } \\
\text { Learning about Beck's cognitive model and the tips on self-monitoring based on this } \\
\text { model }\end{array}$ \\
6 & $\begin{array}{l}\text { Cognitive restructuring, part 2 and } \\
\text { relaxation } \\
\text { Effective problem-solving technique }\end{array}$ & $\begin{array}{l}\text { Learning about cognitive restructuring skills and relaxation skills } \\
\text { Learning about problem-solving methods }\end{array}$ \\
\hline
\end{tabular}




\section{Module 1: mechanism of stress}

In the first module, participants will learn about a transactional stress model [37]. According to the model, an individual's reaction to stressors, in part, depends on their own appraisal of the stressor. Learning the transactional stress model becomes the basis for understanding what they are experiencing in a stressful situation. Ms. Rino, a clinical psychologist, will teach the relationship between a stressor and the stress reaction to the nurse "Ms. Abe," another character. Homework for this module asks the participants to list their own stressors and stress reactions.

\section{Module 2: tips on self-case formulation using a CB model}

In this module, participants will learn about a cognitive behavioral (CB) model and receive tips on self-case formulation. Case formulation is a method to understand the client's problem individually [38]. Case formulation using a CB model has two main advantages. First, it helps us determine what the client experiences in stressful situations. Case formulation using this model considers the client's problem from five perspectives (situation, thoughts, emotions, physical feelings, and behavior) [39] to facilitate understanding of the client's problem. Another advantage is that it helps us consider effective solutions to vicious patterns that afflict a client. Ms. Rino will explain the method of case formulation using an example of a nurse with occupational stress. Additionally, she will offer tips on self-case formulation. This module's homework includes self-case formulation concerning a participant's stressful event.

\section{Module 3: tips to increase your activity}

In this module, participants will learn about a behavioral activation (BA) technique. BA is a process to increase pleasurable and rewarding activities [40]. A meta-analysis reported that BA improved depressive symptoms among clinical populations $(\mathrm{SMD}=0.74,95 \%$ confidence interval $[\mathrm{CI}]=0.56-$ 0.91) [41]. Another meta-analysis reported that BA, often combined with CBT interventions, prevents depression in non-clinical populations [42]. In the CB model, thoughts, emotions, physical feelings, and behavior interact. Increasing pleasurable and rewarding activities can reduce negative thoughts and moods and improve physical responses to stress. Because pleasurable and rewarding activities differ across individuals, BA includes a process of developing and individually suited activity schedule. Ms. Rino will explain the theory of BA, and she will demonstrate how to plan pleasurable and rewarding activities. This module's homework asks the participants to develop and schedule an activity.

\section{Module 4: cognitive restructuring part 1}

In this module, participants will first learn about the "cognitive ABC (Activating/Actual event, Belief, and Consequence) model" [43]. The model explains the perception of an event as stressful rather than the event itself induces stress reactions. Ms. Rino will use a common stressful situation in the nursing field as an example to explain the model. By learning about the model, participants will notice that if their thoughts change, their emotions can improve. Subsequently, they will learn about Beck's cognitive model and receive tips on self-monitoring based on this model. The model premises that dysfunctional schemas shape an individual's automatic thoughts, thus influencing the individuals' emotions [44]. Participants will recognize the importance of identifying their own automatic thoughts that cause a negative mood. This module's homework includes an exercise to monitor participants' own automatic thoughts and emotions in a stressful situation.

\section{Module 5: cognitive restructuring part 2 and relaxation}

In this module, participants will learn about a cognitive restructuring skill useful in cognitive therapy. This cognitive restructuring skill is a major standard component of CBT [45]. A meta-analysis showed that cognitive therapy significantly reduced depressive symptoms among patients with major depressive disorder compared with no intervention [46]. In addition, a previous Cochrane review reported that CBT resulted in significant improvements in stress-related outcomes, including depressive symptoms, compared to no intervention among nurses $(\mathrm{SMD}=0.34)$ [4]. Ms. Rino will teach participants how to change an automatic maladaptive thought into a rational thought. Participants will also learn about a relaxation technique. A Cochrane review reported that relaxation significantly improved stress-related outcomes, including depressive symptoms, compared to no intervention among nurses $(\mathrm{SMD}=0.59)$ [4]. In this module's homework, participants will apply a cognitive restructuring skill to address their stressful situations.

\section{Module 6: effective problem-solving skill}

In this module, participants will learn a problem-solving skill. A meta-analysis reported that problem-solving was effective in the treatment of depression in adults, with Cohen's $d=0.83$ (95\% CI: 0.45 to 1.21 ) compared with control conditions [47]. Additionally, a previous Cochrane review reported that a problem-solving skill, often included in CBT interventions, was effective in the primary prevention of stress-related outcomes, including occupational stress and depressive symptoms, among healthcare workers [4]. Theoretically, a rational problem-solving skill must (1) clarify a problem to be addressed, (2) generate potential solutions to cope with the problem, (3) evaluate each solution, (4) implement selected solutions, and assess the result [48]. Ms. Rino will teach the nurse, Ms. Abe, each step in the process. This module's homework includes a problem-solving exercise involving stressful situations that participants encounter in their workplace or everyday life. 


\section{Intervention group}

Participants in the intervention group will be expected to complete one module a week. Each week, one module will be delivered, and all modules will be delivered in 6 weeks after the program starts. Every Monday, the research center will send a reminder email to those who have not completed each module.

\section{Control group}

Participants in the control group will receive the iCBT program after the six-month follow-up. The participants in the intervention group and the control group will be able to undergo treatment as usual (TAU), such as stress management education or training provided by the nursing association. Few lectures address the knowledge and skills involved in CBT in such education or training. After the six-month follow-up, the iCBT program will be provided to participants in the control group for 9 weeks upon their request.

\section{Outcomes}

Table 2 shows an assessment schedule of this study's outcome measures. All primary and secondary outcomes except for the onset of MDE will be assessed at the baseline (T1), three-month follow-up (T2; immediately after the completion of the intervention), and six-month follow-up (T3). The onset of MDE will be assessed at the baseline and six-month follow-up. Only participants in the intervention group will be assessed regarding the usability of the iCBT program and their satisfaction with it at the three-month follow-up. Information contamination will be assessed at the three- and six-month follow-ups. All data will be collected using web-based self-report questionnaires. At the three- and six-month follow-ups, the research center will send at least two reminder emails to those who have not responded to the questionnaires.

\section{Primary outcome Depressive symptoms}

The Beck Depression Inventory-II (BDI-II) is a self-report inventory that evaluates depression severity. BDI-II includes 21 items measuring depressive symptoms, such as sadness, pessimism, suicidal thoughts or wishes, tiredness or fatigue, loss of energy, and loss of pleasure $[49,50]$. Each item is scored on a four-point scale ranging from 0 to 3 . Higher scores indicate higher depression severity. The Japanese version of the BDI-II developed by Kojima et al. (2002) has shown the same internal reliability and validity as the original version [51].

\section{Secondary outcomes Incidence of MDE}

The onset of MDE will be assessed using the online version of the Japanese WHO-CIDI v 3.0 depression section based on the DSM, Fourth Edition, Text Revision (DSM-IV-TR) criteria [23, 52]. CIDI is a structured diagnostic interview, administered by trained individuals to the general population [53]. The Japanese version of the WHO-CIDI v 3.0 was developed by Kawakami et al. (2005), and its validity in diagnosing MDE has been confirmed [23]. The online version asks respondents the same set of questions using the skip logic of the depression section. A computer program

Table 2 Assessment schedule of the outcome measures for this study

\begin{tabular}{|c|c|c|c|c|}
\hline Measurement & Aim & $\begin{array}{l}\text { Baseline } \\
\text { (T1) }\end{array}$ & $\begin{array}{l}3-M F / \\
U \\
\text { (T2) }\end{array}$ & $\begin{array}{l}\text { 6-MF/ } \\
U \\
\text { (T3) }\end{array}$ \\
\hline \multicolumn{5}{|l|}{ Primary outcome } \\
\hline $\mathrm{BDI}-\mathrm{II}$ & Severity of depression & $x$ & $x$ & $x$ \\
\hline \multicolumn{5}{|l|}{ Secondary outcomes } \\
\hline CIDI & The onset of MDE & $x$ & & $x$ \\
\hline JCQ & Psychosocial work environment & $x$ & $x$ & $x$ \\
\hline UWES & Work engagement & $x$ & $x$ & $x$ \\
\hline $\mathrm{HPQ}$ & Work performance & $x$ & $x$ & $x$ \\
\hline Sick leave days & Sick leave days during the past 3 months & $x$ & $x$ & $x$ \\
\hline Intention to leave & Intention to leave their organization & $x$ & $x$ & $x$ \\
\hline $\begin{array}{l}\text { Knowledge and self- } \\
\text { efficacy }\end{array}$ & $\begin{array}{l}\text { Current knowledge of and self-efficacy in relation to the five components of the CBT } \\
\text { program }\end{array}$ & $x$ & $x$ & $x$ \\
\hline \multicolumn{5}{|l|}{ Process evaluation } \\
\hline Usability and satisfaction & Usability and satisfaction of the iCBT program & & $x$ & \\
\hline \multicolumn{5}{|l|}{ Others } \\
\hline Contamination & Contamination of information & & $x$ & $x$ \\
\hline
\end{tabular}

Note: BDI-II Beck Depression Inventory II, CIDI WHO-Composite International Diagnostic Interview, MDE Major Depressive Episode, JCQ Job Content Questionnaire, UWES Utrecht Work Engagement Scale, HPQ WHO Health and Work Performance Questionnaire, CBT Cognitive Behavioral Therapy 
with an algorithm specific to WHO-CIDI v 3.0 automatically diagnoses MDE. The online version has been shown to be consistent in the clinical diagnosis of MDE, [54] and the reliability of this version has been confirmed in a 1-year test-retest survey [55]. While WHO-CIDI v 3.0 was originally designed to generate a diagnosis based on the DSM-IVTR criteria, its instrument can also generate a diagnosis of MDE according to DSM-5 criteria [56].

\section{Psychosocial work environment}

Psychosocial work environment will be evaluated using the Japanese version of the Job Content Questionnaire (JCQ) $[57,58]$. The JCQ is a 22-item self-administered instrument designed to measure the psychosocial environment in the workplace, based on Karasek's Job Demands-Control model [59] and the Demand-ControlSupport model [60]. The JCQ scales consist of psychological demands (5 items), job control (9 items), supervisor support (4 items), and coworker support (4 items). Each item is scored on a scale from 1 (strongly disagree) to 4 (strongly agree). The reliability and validity of the Japanese version of the JCQ are acceptable [57].

\section{Work engagement}

Work engagement is a new concept from a positive perspective of mental health. Work engagement will be measured using the short form of the Japanese version of the Utrecht Work Engagement Scale (UWES-J) [61, 62]. UWES-J consists of nine items measuring three subscales (i.e., vigor, dedication, absorption). Each item is assessed on a seven-point scale from 0 (never) to 6 (always). The total score is calculated from all nine items. The reliability and validity of the UWES-J have been established [62].

\section{Work performance}

Work performance will be evaluated using one item of the WHO Health and Work Performance Questionnaire (HPQ) [63]. The HPQ is a self-report measure designed to estimate the workplace costs of health problems. In this study, participants will be asked to rate their overall work performance during the past 4 weeks. Items are scored on an 11-point scale ranging from 0 (worst possible performance) to 10 (best possible performance). A high score indicates a high degree of work performance.

\section{Sick leave days during the past three months}

Participants will be asked to report their number of sick leave days during the past 3 months.

\section{Intention to leave}

Intention to leave will be measured using the Japanese version of the four-item scale used by Geurts, Schaufeli, and De Jonge [64, 65]. The scale is a self-report inventory assessing the extent to which respondents expressed an intention to leave their organization during the last month. Each item is scored on a scale from 1 (strongly disagree) to 5 (strongly agree). Higher scores indicate greater intention to leave. Reliability and validity of the Japanese version have not yet been determined.

\section{Knowledge and self-efficacy}

Participants will be asked to rate their current knowledge of and self-efficacy in the five CBT components of the program (stress management, behavioral activation, cognitive reconstruction, relaxation training, and problem solving). The items assessing knowledge will start with the question, "How much knowledge do you have about..." while the items assessing self-efficacy will start with "How confident are you that you can do..." Each item will be scored on a 5-point scale ranging from 0 (none) to 4 (enough). Higher scores indicate greater knowledge of and self-efficacy in CBT components.

\section{Psychological distress}

Psychological distress will be measured only at baseline using the Japanese version of the K6 [30, 31]. Participants will be stratified at baseline based on K6 score (5 or greater or less than 5). The K6 is a self-report inventory assessing the frequency with which respondents have experienced symptoms of psychological distress during the past 30 days. Each item is scored on a scale from 0 (never) to 4 (all of the time). Higher scores indicate more severe psychological distress [66]. The internal reliability and validity of the Japanese version of the K6 are acceptable [30].

\section{Process evaluation}

\section{Usability of and satisfaction with the iCBT program}

The usability of and satisfaction with the iCBT program will be assessed using original questions. Sample questions include, "How easy was it to understand the program?" and "How satisfied were you with the program as a whole?" Each item will be scored on a 5-point scale ranging from 0 (nоnе) to 4 (enough). In addition, participants will be asked which device they used most frequently.

\section{The number and types of modules and homework}

The number and types of modules and homework will be evaluated using the e-learning system records.

\section{Contamination of information}

Contamination of information will be assessed at threeand six-month follow-ups through one original question answered on a dichotomous yes/no scale: "During the past three months, have you received information on stress management from your colleagues who used this study's iCBT program?" 


\section{Demographic characteristics}

Demographic data, including age, gender, marital status, education, employment status, position, years of experience as a nurse, and overtime hours during the past month will be collected.

\section{Sample size calculation}

This study's iCBT program will include mental health professionals' support. Previous meta-analyses of iCBT aimed at improving depressive symptoms among workers, both with or without supports, yielded effect sizes of $0.16-0.25$ [19, 20]. Programs with supports have been reported to be more effective compared to self-guided iCBT programs $[15,20]$. To detect a small effect size of 0.2 or greater at an alpha error probability of 0.05 and a power $(1-\beta)$ of 0.80 using G*Power V.3.1.9.2 [67], a sample size of 394 participants per arm would be required. Anticipating a dropout rate of $25 \%$, the necessary sample size would be 525 participants per arm.

\section{Randomization}

After the baseline survey, eligible participants will be randomly assigned either to the intervention or control group using stratified permuted-block randomization, with a random block size of two. Participants will be stratified based on K6 score (5 or greater or less than 5) at baseline survey and worksite. The intervention effect may differ according to the severity of the psychological distress at baseline. The K6 cut-off score will be based on the optimal cut-off value (i.e., 4/5), which was used to separate patients with mood disorders in a previous study [66]. An independent biostatistics researcher will generate a stratified permuted-block random table. This table will be password-protected and concealed to other researchers. The researcher (KK) will carry out enrollment and another independent researcher will conduct allocation. During the random assignment, only the researcher will be able to access the stratified permuted-block random table.

\section{Statistical methods Clinical efficacy}

For primary and secondary outcomes, except the onset of MDE, a mixed model for repeated measures conditional growth model analysis will be conducted on an intention-to-treat basis. We will analyze a group (intervention and control) * time (baseline and three- and sixmonth follow-ups) interaction as an indicator of intervention effect. Missing values will be imputed by applying maximum likelihood estimation using the MIXED procedure. As the surveys will be conducted via the Internet, missing values will not occur unless the participants do not answer the questions. Cohen's $d$ between groups and 95\% confidence intervals will be calculated at each assessment term to assess effect size. The values of $0.2,0.5$, and 0.8 are generally interpreted as small, medium, and large effects, respectively [68]. In addition, we will also calculate the Number Needed to Treat (NNT) because of the difficulty of interpreting the effect size from a clinical perspective. The NNT is the number of persons who must be treated to yield a difference in the onset of an event in one person compared to the control group. In this study, the NNT to alleviate depressive symptoms to achieve improvement in one individual from subthreshold depression will be calculated. Based on the cut-off BDI-II score, this improvement was defined as a change from a score of 14 or more to a score of 13 or less.

Regarding the onset of MDE, a survival analysis will be performed to compare the intervention and the control groups in terms of their mean survival time without MDE to evaluate the effectiveness of the intervention. The survival time will be calculated for each participant as months from the baseline survey to the onset of MDE or the end of six-month follow-up survey. The cumulative incidence ratio of MDE and event-free survival ratio at the six-month follow-up will be estimated using the Kaplan-Meier method. To test the difference in survival probability between the intervention and the control group, a log-rank test will be performed.

\section{Subgroup analysis}

Since the effect of the iCBT program may differ according to specific population, the effect will also be analyzed by subgroups. Participants will be divided according to their initial severity of psychological distress. We will treat high/low psychological distress (i.e., participants who scored 5 or more on $\mathrm{K} 6$ at baseline) as one stratification factor and perform subgroup analysis. In addition, newly graduated nurses (i.e., less than 1 year of nursing experience) have been reported to have higher depressive symptoms compared to veteran nurses $[7,69,70]$. We will treat participants with more/less than 1 year of nursing experience as another stratification factor and conduct subgroup analysis.

\section{Data monitoring}

A data monitoring committee (DMC) will consist of at least one independent member. The trial will be checked by the DMC. After randomizing the participants, the DMC will meet every 3 months to review a report created by the researchers to monitor the progress of recruitment and data collection (e.g., completion rates for each follow-up).

\section{Data confidentiality}

After the surveys, the collected data will be transferred to a password-locked stand-alone PC. The PC will be kept in a locked research room at the Department of Psychiatric Nursing in the Graduate School of Medicine at the University of Tokyo. The collected data will be saved as linkable 
anonymous data with sequentially allocated numbers. The researchers will be able to access the data.

\section{Dissemination of research findings}

The findings of this study will be published in peerreviewed international journals. We will also present the results and findings at related research conferences, academic symposiums, and seminars.

\section{Discussion}

The greatest strength of this study is that it is the first RCT, to the best of our knowledge, to evaluate the effect of an iCBT program on improving depressive symptoms among nurses. If successful, the iCBT program for nurses could be a promising tool to improve depressive symptoms as a primary prevention. Additionally, this study also aims to add to the evidence on the effect of iCBT programs on positive health outcomes (i.e., work engagement and work performance) among nurses.

Nevertheless, this study has some limitations. All data, including MDE measurement, will be measured by self-report, which may be affected by situational factors at work and participants' perceptions. In addition, due to limited resources and funding, the participants will not be able to submit their homework via smartphone, which may limit the effect of our intervention program. Moreover, there is the possibility that participants in the control group will obtain information about the iCBT program from participants in the intervention group in the same ward or workplace. This contamination may weaken the intervention effect.

\section{Abbreviations \\ BA: behavioral activation; BDI-II: Beck Depression Inventory-II; CB: cognitive behavioral; CBT: cognitive behavioral therapy; Cl: confidence interval; DMC: data monitoring committee; DSM-IV-TR: DSM, Fourth Edition, Text Revision; EAP: Employee Assistance Program; HPQ: WHO Health and Work Performance Questionnaire; iCBT: Internet-based cognitive behavioral therapy; JCQ: Job Content Questionnaire; MDE: major depressive episode; NNT: Number Needed to Treat; RCT: randomized controlled trial; SMD: standardized mean difference; SPIRIT: Standard Protocol Items: Recommendations for Interventional Trials; TAU: treatment as usual; UMIN: University Hospital Medical Information Network; UMIN-CTR: University Hospital Medical Information Network clinical trial registry; UWES: Utrecht Work Engagement Scale; WHO-CIDI: WHO-Composite International Diagnostic Interview}

\section{Acknowledgements}

The authors wish to thank the members of the Department of Psychiatric Nursing, Mental Health, and Nursing Administration and Advanced Clinical Nursing at The University of Tokyo for their advice in developing iCBT program for nurses.

\section{Authors' contributions}

$\mathrm{KK}, \mathrm{Kl}$, and NK conceived and designed the experiments. KK, Kl, KW, YM, AT, US, NS, MSug, AS, YH, MI, MSud, MT, and NK contributed reagents/materials/ analysis tools. KK wrote the initial draft of the manuscript, and all authors revised and contributed to the final manuscript. All authors read and approved the final manuscript.

\section{Funding}

Not applicable.
Availability of data and materials

Those interested in this study will communicate all outcomes and findings through publications.

\section{Ethics approval and consent to participate}

The Research Ethics Review Board of the Graduate School of Medicine at the University of Tokyo approved the study procedures (11945-(1)). This also covers all other participating hospitals' ethical approval. We prepared a website that contains a full explanation of the purpose and procedures of this study. Before the baseline survey, candidates will be asked to read the explanation in which they will be fully informed that participation in the study is voluntary, and they can refuse to participate or withdraw consent for any reason, which would have no negative consequences for them. In addition, participants will be explained that the findings of this study will be disseminated through publications, presentations, and websites without participants' personal information. If there are any unclear points about the purpose and procedures of this study, the candidate can ask the researcher (KK) questions by an email or a telephone. Participants who will be interested in participating in this study will be asked to check agree option and type their names, hospital names where they are working, and their email addresses to indicate their consent to participate in the study on the website. This consent information will be sent to the research center and preserved.

The study protocol was registered at the UMINCTR (ID = UMIN000033521). In case we need to submit important revisions to the protocol, we will obtain approval to do so from the Research Ethics Review Board of the Graduate School of Medicine at the University of Tokyo and will revise the protocol on the UMINCTR website.

\section{Consent for publication}

Not applicable.

\section{Competing interests}

The authors declare that they have no competing interests.

\section{Author details}

${ }^{1}$ Department of Psychiatric Nursing, Graduate School of Medicine, The University of Tokyo, 7-3-1 Hongo, Bunkyo-ku, Tokyo 113-0033, Japan. ${ }^{2}$ Department of Mental Health, Graduate School of Medicine, The University of Tokyo, 7-3-1 Hongo, Bunkyo-ku, Tokyo 113-0033, Japan. ${ }^{3}$ Department of Mental Health and Psychiatric Nursing, Graduate School of Health Care Sciences, Tokyo Medical and Dental University, 1-5-45 Yushima, Bunkyo-ku, Tokyo 113-8510, Japan. ${ }^{4}$ Department of Keio Research Institute at SFC (Shonan Fujisawa Campus), Keio University SFC, 5322 Endo, Fujisawa-shi, Kanagawa 252-0882, Japan.

Received: 7 November 2018 Accepted: 26 July 2019

Published online: 07 August 2019

\section{References}

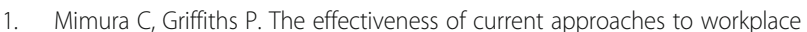
stress management in the nursing profession: an evidence based literature review. Occup Environ Med. 2003;60:10-5.

2. McVicar A. Workplace stress in nursing: a literature review. J Adv Nurs. 2003; 44:633-42.

3. Marcus M, Yasamy MT, van Ommeren M, Chisholm D, Saxena S. Depression: a global public health concern. WHO Dep Ment Heal Subst Abus. 2012;1:6-8.

4. Ruotsalainen $\mathrm{JH}$, Verbeek JH, Mariné A, Consol S. Preventing occupational stress in healthcare workers ( review ) SUMMARY OF FINDINGS FOR THE MAIN COMPARISON. Cochrane Database Syst Rev Prev 2015:

5. Welsh D. Predictors of depressive symptoms in female medical-surgical hospital nurses. Issues Ment Health Nurs. 2009;30:320-6.

6. Letvak S, Ruhm CJ, McCoy T. Depression in hospital-employed nurses. Clin Nurse Spec. 2012;26:177-82.

7. Brandford AA, Reed DB. Depression in registered nurses: a state of the science. Workplace Health Saf. 2016;64:488-511.

8. Baba W, Galperin BL, Lituchy TR. Occupational mental health: a study of work-related depression among nurses in the Caribbean. Int I Nurs Stud. 1999:36:163-9.

9. Innstrand ST, Langballe EM, Falkum E. A longitudinal study of the relationship between work engagement and symptoms of anxiety and depression. Stress Health. 2012;28:1-10. 
10. Letvak S, Ruhm C, Gupta S. Nurses' presenteeism and its effects on selfreported quality of care and costs. Am J Nurs. 2012;112:30-8 quiz 48, 39.

11. Joyce S, Modini M, Christensen H, Mykletun A, Bryant R, Mitchell PB, et al. Workplace interventions for common mental disorders: a systematic metareview. Psychol Med. 2016;46:683-97.

12. Richardson KM, Rothstein HR. Effects of occupational stress management intervention programs: a meta-analysis. J Occup Health Psychol. 2008;13:69-93.

13. van der Klink JJ, Blonk RW, Schene AH, van Dijk FJ. The benefits of interventions for work-related stress. Am J Public Health. 2001;91:270-6.

14. Titov N, Andrews G, Sachdev P. Computer-delivered cognitive behavioural therapy: effective and getting ready for dissemination. F1000 Med Rep. 2010;2:2-5.

15. Spek V, Cuijpers P, Nyklícek I, Riper H, Keyzer J, Pop V. Internet-based cognitive behaviour therapy for symptoms of depression and anxiety: a meta-analysis. Psychol Med. 2007;37:319-28.

16. Ryan C, Bergin M, Chalder T, Wells JS. Web-based interventions for the management of stress in the workplace: focus, form, and efficacy. J Occup Health. 2017;59:215-36.

17. Yung PMB, Fung MY, Chan TMF, Lau BWK. Relaxation training methods for nurse managers in Hong Kong: a controlled study. Int J Ment Health Nurs. 2004;13:255-61.

18. Ball J, Pike G, Cuff C, Mellor-Clark J, Connell J. Working well? Results from the RCN working well survey into the wellbeing and working lives of nurses; 2002.

19. Carolan S, Harris PR, Cavanagh K. Improving employee well-being and effectiveness: systematic review and meta-analysis of web-based psychological interventions delivered in the workplace. J Med Internet Res. 2017;19:1-18.

20. Stratton E, Lampit A, Choi I, Calvo RA, Harvey SB, Glozier N. Effectiveness of eHealth interventions for reducing mental health conditions in employees: a systematic review and meta-analysis. PLoS One. 2017;12:1-23.

21. Hersch RK, Cook RF, Deitz DK, Kaplan S, Hughes D, Friesen MA, et al. Reducing nurses' stress: a randomized controlled trial of a web-based stress management program for nurses. Appl Nurs Res. 2016;32:18-25.

22. Chan A, Tetzlaff JM, Altman DG, Laupacis A, Gøtzsche PC, Krleža-Jerić K, et al. SPIRIT 2013 statement: defining standard protocol items for clinical trials. Ann Intern Med. 2013;158:200-7.

23. Kawakami N, Takeshima T, Ono Y, Uda H, Hata Y, Nakane Y, et al. Twelvemonth prevalence, severity, and treatment of common mental disorders in communities in Japan: preliminary finding from the world mental health Japan survey 2002-2003. Psychiatry Clin Neurosci. 2005;59:441-52.

24. Kuribayashi K, Imamura K, Tokita M, Shimazu A, Kawakami N. Effects of an internet-based cognitive behavioral therapy (iCBT) intervention on depressive symptoms, dysfunctional attitude, and work engagement among new graduate nurses: a pilot study. In: The 22th annual meeting of Japan Society for Occupational Mental Health. Tokyo: Japan Society for Occupational Mental Health; 2015. p. 144.

25. Imamura K, Kawakami N, Furukawa TA, Matsuyama Y, Shimazu A, Umanodan R, et al. Effects of an internet-based cognitive behavioral therapy (iCBT) program in manga format on improving subthreshold depressive symptoms among healthy workers: a randomized controlled trial. PLoS One. 2014;9:e97167.

26. Kishi Y, Matsumura T, Murishige N, Kodama Y, Hatanaka N, Takita M, et al. Internetbased survey on medical manga in Japan. Health Commun. 2011;26:676-8.

27. Hutchinson $\mathrm{KH}$. An experiment in the use of comics as instructional material. J Educ Sociol. 1949;23:236-45.

28. Perini S, Titov N, Andrews G. Clinician-assisted internet-based treatment is effective for depression: randomized controlled trial. Aust N Z J Psychiatry. 2009:43:571-8

29. Merry SN, Stasiak K, Shepherd M, Frampton C, Fleming T, Lucassen MFG The effectiveness of SPARX, a computerised self help intervention for adolescents seeking help for depression: randomised controlled noninferiority trial. BMJ. 2012;344:1-16.

30. Furukawa TA, Kawakami N, Saitoh M, Ono Y, Nakane Y, Nakamura Y, et al. The performance of the Japanese version of the K6 and K10 in the world mental health survey Japan. Int J Methods Psychiatr Res. 2008;17:152-8.

31. Kessler RC, Andrews G, Colpe L, Hiripi E, Mroczek DK, Normand SLT, et al. Short screening scales to monitor population prevalences and trends in non-specific psychological distress. Psychol Med. 2002;32:959-76.

32. Spurgeon JA, Wright JH. Computer-assisted cognitive-behavioral therapy. Curr Psychiatry Rep. 2010;12:547-52

33. Morrison LG, Yardley L, Powell J, Michie S. What Design Features are Used in Effective eHealth Interventions? A Review using Techniques from Critical Interpretative Synthesis. 2012;18:137-44.
34. Waller R, Gilbody S. Barriers to the uptake of computerized cognitive behavioural therapy : a systematic review of the quantitative and qualitative evidence. 2018;705-12.

35. Hilvert-Bruce Z, Rossouw PJ, Wong N, Sunderland M, Andrews G. Adherence as a determinant of effectiveness of internet cognitive behavioural therapy for anxiety and depressive disorders. Behav Res Ther. 2012;50:463-8.

36. Eysenbach G. The law of attrition. J Med Internet Res. 2005;7:1-9.

37. Lazarus R, Folkman S. Coping and adaptation. The handbook of behavioral medicine 1984

38. Adams H. Further clarifications of case formulation. Behav Ther. 1996;19:78.

39. Padesky C, Greenberger D. Clinician's guide to mind over mood. New York: Guilford Press; 1995.

40. Martell CR, Dimidjian S, Herman-Dunn R. Behavioral activation for depression: a clinician's guide. New York: Guilford Press; 2010.

41. Ekers D, Webster L, Van Straten A, Cuijpers P, Richards D, Gilbody S. Behavioural activation for depression; an update of meta-analysis of effectiveness and sub group analysis. PLoS One. 2014;9.

42. Cuijpers P, Muñoz RF, Clarke GN, Lewinsohn PM. Psychoeducational treatment and prevention of depression: the "coping with depression" course thirty years later. Clin Psychol Rev. 2009;29:449-58.

43. Ellis A. Humanistic psychotherapy: the rational-emotive approach. New York: Julian Press; 1973.

44. Beck A. Depression: clinical, experimental, and theoretical aspects. New York: Hoeber Medical Division; 1967.

45. Beck AT, Dozois DJ. Cognitive therapy: current status and future directions. Annu.Rev.Med. 2011;62:397-409.

46. Jakobsen JC, Hansen JL, Storebø OJ, Simonsen E, Gluud C. The effects of cognitive therapy versus "no intervention" for major depressive disorder. PLoS One. 2011;6:e28299.

47. Cuijpers P, Andersson G, Donker T, Van Straten A. Psychological treatment of depression: results of a series of meta-analyses. Nord J Psychiatry. 2011;65:354-64.

48. D'Zurilla TJ, Goldfried MR. Problem solving and behavior modification. J Abnorm Psychol. 1971;78:107-26.

49. Beck A, Steer R, Brown G. BDI-II, Beck depression inventory: manual. Vi. San Antonio, Tex. Boston: Psychological Corp; 1996.

50. Hiroe T, Kojima M, Yamamoto I, Nojima S, Kinoshita Y, Hashimoto N, et al. Gradations of clinical severity and sensitivity to change assessed with the Beck depression inventory-II in Japanese patients with depression. Psychiatry Res. 2005;135:229-35.

51. Kojima M, Furukawa TA, Takahashi H, Kawai M, Nagaya T, Tokudome S. Cross-cultural validation of the Beck depression inventory-II in Japan. Psychiatry Res. 2002;110:291-9.

52. Kessler RC, Ustün TB. The world mental health (WMH) survey initiative version of the World Health Organization (WHO) composite international diagnostic interview (CIDI). Int J Methods Psychiatr Res. 2004;13:93-121.

53. Robins LN, Wing J, Wittchen HU, Helzer JE, Babor TF, Burke J, et al. The composite international diagnostic interview. An epidemiologic Instrument suitable for use in conjunction with different diagnostic systems and in different cultures. Arch Gen Psychiatry. 1988;45:1069-77.

54. Inoue A, Kawakami N, Tsuno K, Tomioka K, Nakanishi M. Organizational justice and major depressive episodes in Japanese employees: a crosssectional study. J Occup Health. 2013;55:47-55.

55. Shimoda H, Inoue A, Tsuno K, Kawakami N. One-year test-retest reliability of a Japanese web-based version of the WHO composite international diagnostic interview (CIDI) for major depression in a working population. Int J Methods Psychiatr Res. 2015;24:204-12.

56. Association AP. Diagnostic and statistical manual of mental disorders, (DSM$5^{\circledR}$ ). American Psychiatric Pub; 2013.

57. Kawakami N, Kobayashi F, Araki S, Haratani T, Furui H. Assessment of job stress dimensions based on the job demands- control model of employees of telecommunication and electric power companies in Japan: reliability and validity of the Japanese version of the job content questionnaire. Int J Behav Med. 1995;2:358-75.

58. Karasek R, Brisson C, Kawakami N, Houtman I, Bongers P, Amick B. The job content questionnaire (JCQ): an intsrument for internationally comparative assessment of psychsocial job characteristics. J Occup Health Psychol. 1998; 3:322-55.

59. Karasek RA. Job demands, job decision latitude, and mental strain: implications for job redesign. Adm Sci Q. 1979;24:285.

60. Johnson JV. The impact of workplace social support, job demands and work control upon cardiovascular disease in Sweden. Diss Abstr Int. 1986;47:591. 
61. Schaufeli WB. The measurement of work engagement with a short questionnaire: a cross-National Study. Educ Psychol Meas. 2006;66:701-16.

62. Shimazu A, Schaufeli WB, Kosugi S, Suzuki A, Nashiwa H, Kato A, et al. Work engagement in Japan: validation of the Japanese version of the Utrecht work engagement scale. Appl Psychol. 2008:57:510-23.

63. Kessler RC, Barber C, Beck A, Berglund P, Cleary PD, McKenas D, et al. The World Health Organization health and work performance questionnaire (HPQ). J Occup Environ Med. 2003;45:156-74.

64. Tsuno K, Kawakami N, Shimazu A, Shimada K, Inoue A, Leiter MP. Workplace incivility in Japan: reliability and validity of the Japanese version of the modified work incivility scale. J Occup Health. 2017;59:237-46.

65. Geurts S, Schaufeli W, De Jonge J. Burnout and intention to leave among mental health-care professionals: a social psychological approach. J Soc Clin Psychol. 1998;17:341-62.

66. Sakurai K, Nishi A, Kondo K, Yanagida K, Kawakami N. Screening performance of K6/K10 and other screening instruments for mood and anxiety disorders in Japan. Psychiatry Clin Neurosci. 2011;65:434-41.

67. Faul F, Erdfelder E, Lang A-G, Buchner A. G*power 3: a flexible statistical power analysis program for the social, behavioral, and biomedical sciences. Behav Res Methods. 2007;39:175-91.

68. Cohen J. A power primer. Psychol Bull. 1992;112:155-9.

69. Feng R-F, Tsai Y-F. Socialisation of new graduate nurses to practising nurses. J Clin Nurs. 2012;21:2064-71.

70. Theisen JL, Sandau KE. Competency of new graduate nurses: A review of their weaknesses and strategies for success. J Contin Educ Nurs. 2013;44:406-14.

\section{Publisher's Note}

Springer Nature remains neutral with regard to jurisdictional claims in published maps and institutional affiliations.

Ready to submit your research? Choose BMC and benefit from:

- fast, convenient online submission

- thorough peer review by experienced researchers in your field

- rapid publication on acceptance

- support for research data, including large and complex data types

- gold Open Access which fosters wider collaboration and increased citations

- maximum visibility for your research: over $100 \mathrm{M}$ website views per year

At BMC, research is always in progress.

Learn more biomedcentral.com/submissions 